\title{
Bullous pemphigoid associated with psoriasis: a good response to methotrexate*
}

\author{
Pablo Vargas ${ }^{1}$, Paula Giacaman², Javier Fernández², Claudia Morales ${ }^{3}$
}

DOI: http:/ / dx.doi.org/10.1590/abd1806-4841.20198032

\begin{abstract}
Psoriasis has been associated with various autoimmune diseases, however, its relation to bullous diseases is infrequent. Of these, bullous pemphigoid appears as the main associated entity, even though both conditions differ considerably in demographic and clinical aspects. We report the case of a 42-year-old female patient, with long-standing psoriasis who consulted due to the exacerbation of psoriatic plaques associated with generalized bullous lesions on the skin and oral mucosa, with one-week duration. With clinical signs and histopathological findings compatible with bullous pemphigoid associated with psoriasis, we decided to treat her with methotrexate $10 \mathrm{mg}$ a week. The patient had an excellent response after two months of treatment.
\end{abstract}

Keywords: Pemphigoid, bullous; Psoriasis; Methotrexate

\section{INTRODUCTION}

Psoriasis (PSO) is a chronic inflammatory disease with a worldwide prevalence of 2 to $4 \% .{ }^{1}$ It has been associated with various autoimmune diseases, such as inflammatory bowel disease, celiac disease, among others, being infrequent its association with bullous diseases. ${ }^{2}$ Bullous pemphigoid (BP) is the most frequent blistering dermatosis and the most associated with PSO, with about 50 cases reported in the literature since 1929.3,4 The pathogenic mechanisms of this association remain unknown, considering that both conditions have well-known demographic, clinical, histopathological and therapeutic differences.

We present the case of a woman with long-standing PSO associated with BP of recent onset.

\section{CASE REPORT}

A 42-year-old female patient had a history of PSO since 17 years of age, without other co-morbidities, under irregular treatment with high-potency topical corticosteroids. She consulted due to oneweek course of generalized bullous lesions on previously healthy skin and oral mucosa, associated with extension of psoriatic plaques on the trunk and extremities, without triggering factors like phototherapy, drugs or sunlight exposure. Physical examination revealed tense and confluent blisters on the back of hands, palms and soles with serous content, erosions on the oral mucosa and extensive desquamative erythematous plaques on the forearms, thighs, legs and lower back (Figure 1). A skin biopsy was performed (3 samples), which revealed psoriasiform dermatitis with parakeratosis, intra-

\footnotetext{
Received 26 December 2017.

Accepted 12 April 2018.

* Work conducted at the San Jose Hospital, Santiago, Chile.

Financial support: None.

Conflict of interest: None.

Department of Dermatology, Faculty of Medicine, University of Chile, Santiago, Chile.

Dermatology Service, San Jose Hospital, Santiago, Chile.

Pathology Service, University of Chile Clinical Hospital, Santiago, Chile.
}

MAILING ADDRESS:

Pablo Vargas Mora

E-mail: pablovargas.med@gmail.com

C2019 by Anais Brasileiros de Dermatologia 
corneal pustule, hypogranulosis, dilated dermal capillaries with predominantly polymorphonuclear moderate superficial inflammatory infiltrate with the presence of a subepidermal blister with neutrophils and eosinophils in one of the samples. In direct immunofluorescence, IgG and C3 deposits with linear pattern were found in the dermoepidermal junction, compatible with BP (Figure 2). Indirect immunofluorescence was not made, due to unavailability.

Treatment was started with methotrexate $10 \mathrm{mg}$ weekly, associated with folic acid $5 \mathrm{mg}$ weekly and medium-potency topical corticosteroids.
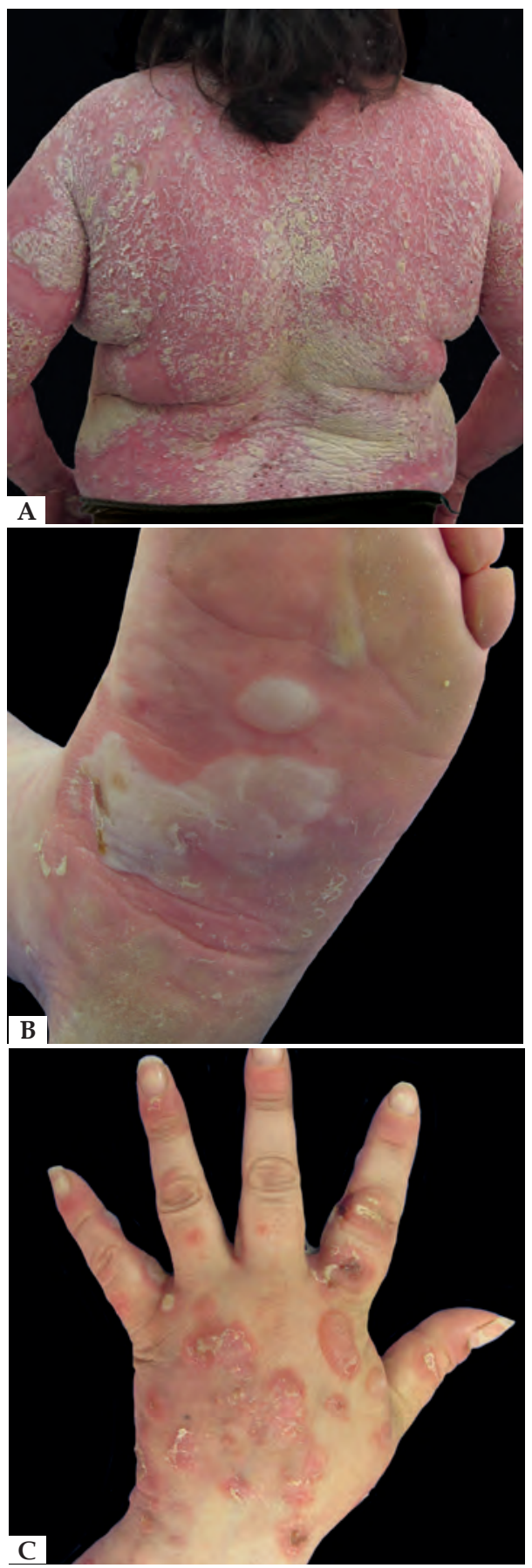

Figure 1:

A - Extensive desquamative erythematous plaques; B - Tense and confluent blisters on the sole; C - Tense and confluent blisters on the back of hand
After 2 months of treatment, the patient showed marked improvement, with remission of the bullous lesions and scarce erythematous desquamative lesions on the dorsal region, maintaining no bullous lesions or psoriatic plaques in the fourth month of follow-up (Figure 3).

\section{DISCUSSION}

The association of PSO and BP is infrequent, with few reports in the literature to allow the estimation of the real frequency in which this association occurs. It is estimated to be around $2.1 \%$ and $5.3 \%$ in Taiwanese and Chinese populations, respectively. ${ }^{5,6} \mathrm{In}$ Latin America, there are only reports of isolated cases. In the larg-

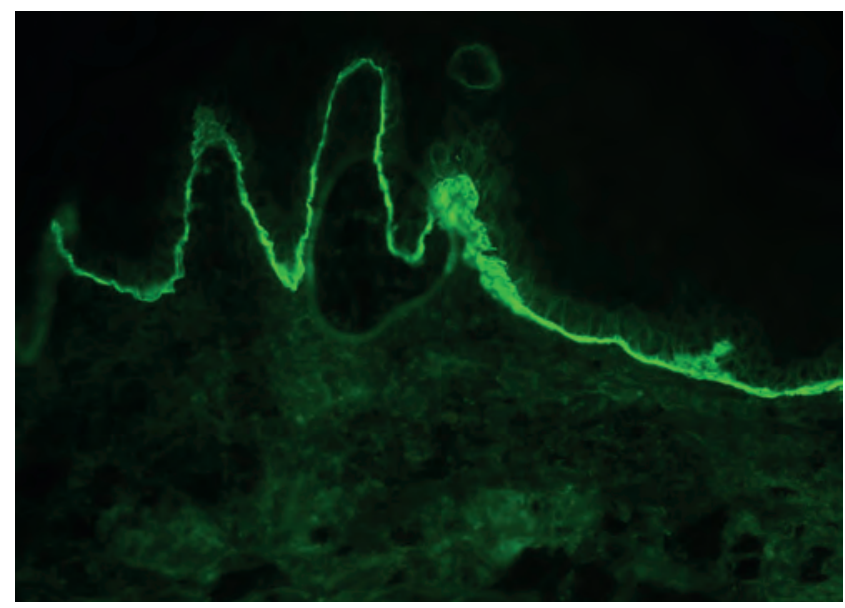

Figure 2: Direct immunofluorescence: $\mathrm{C} 3$ deposits with a linear pattern in the dermoepidermal junction

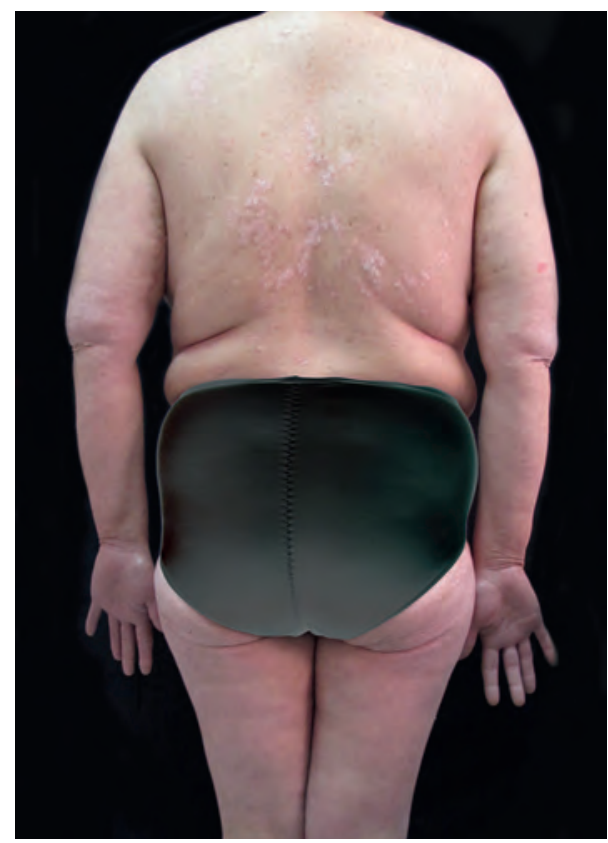

Figure 3: After two months of treatment 
est case series that included 145 patients with bullous diseases and $\mathrm{PSO}$, it was found that $63 \%$ of these corresponded to BP, being more frequent in men $(82.1 \%)$ with ages between 50 to 80 years. Blistering lesions are described in both healthy skin and skin with psoriatic plaques. In most cases, the PSO precedes BP in several years, with an average interval of 15 to 25 years. ${ }^{3,6,7}$ Regarding the proposed pathogenic mechanisms, it is described that for unknown reasons there would be an alteration of the immune response mediated by T lymphocytes, in which antibodies directed against stratum corneum and granulosum structures present in psoriasis could attack the basement membrane. On the other hand, electron microscopy has shown damage to the basement membrane in patients with PSO, generating alterations in antigenicity, with subsequent exposure and development of autoantibodies. ${ }^{8}$ Anti-p200 pemphigoid or anti-laminin-gamma-1 pemphigoid, described just over 20 years ago, with great clinical and histopathological similarity to BP, emerges as a bullous disease of frequent association with PSO, mainly in the Japanese population, which suggests that a high proportion of the cases of pemphigoid and PSO would correspond to this entity. ${ }^{9}$ The lack of availability of immunoassay techniques for the detection of anti-p200 could lead to an overestimation of the coexistence between PSO and BP. One of the greatest challenges of this association is management, with methotrexate being the most used therapeutic option with good response, as presented in this case. As second line, have been reported systemic corticosteroids, cyclosporine, azathioprine, dapsone and erythromycin with etretinate. ${ }^{4,10}$ The present case is reported given the uncommon association between both conditions, especially in the Latin population, in addition to highlighting the rapid and favorable response of both conditions to methotrexate.]

\section{REFERENCES}

1. Parisi R, Symmons DP, Griffiths CE, Ashcroft DM; Identification and Management of Psoriasis and Associated ComorbidiTy (IMPACT) project team. Global epidemiology of psoriasis: a systematic review of incidence and prevalence. $J$ Invest Dermatol. 2013;133:377-85.

2. Oliveira Mde F, Rocha Bde O, Duarte GV. Psoriasis: classical and emerging comorbidities. An Bras Dermatol. 2015;90:9-20.

3. Wilczek A, Sticherling M. Concomitant psoriasis and bullous pemphigoid: coincidence or pathogenic relationship? Int J Dermatol. 2006;45:1353-7.

4. Rao R, Gupta A, Yunis F, Handettu S, Chandrashekar B. Coexistence of psoriasis with bullous pemphigoid. Indian Dermatol Online J. 2012;3:119-21.

5. Zhang LM, Wu J, Xiao T, Jin GY, Li JH, Geng L, et al. Treatment and mortality rate of bullous pemphigoid in China: a hospital-based study. Eur J Dermatol. 2013 ;23:94-8.
6. Ohata C, Ishii N, Koga H, Fukuda S, Tateishi C, Tsuruta D, et al. Coexistence of autoimmune bullous diseases (AIBDs) and psoriasis: A series of 145 cases. J Am Acad Dermatol. 2015;73:50-5.

7. Kridin K, Bergman R. Association between bullous pemphigoid and psoriasis: A case-control study. J Am Acad Dermatol. 2017;77:370-2.

8. Kirtschig G, Chow ET, Venning VA, Wojnarowska FT. Acquired subepidermal bullous diseases associated with psoriasis: a clinical immunopathological and immunogenetic study. Br J Dermatol. 1996;135:738-45.

9. Yasuda H, Tomita Y, Shibaki A, Hashimoto T. Two cases of subepidermal blistering disease with anti-p200 or 180-kD bullous pemphigoid antigen associated with psoriasis. Dermatology. 2004;209:149-55.

10. Iskandarli M, Gerceker Turk B, Yaman B, Ozturk G. Pemphigoid diseases as a sign of active psoriasis: A case report and brief review. Dermatology. 2015;231:319-21.

Approval of the final version of the manuscript; Elaboration and writing of the manuscript; Obtaining, analyzing and interpreting the data; Effective participation in research orientation; Intellectual participation in propaedeutic and/or therapeutic conduct of the cases studied; Critical review of the literature; Critical review of the manuscript

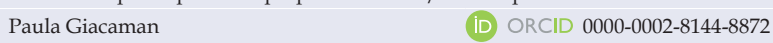

Approval of the final version of the manuscript; Elaboration and writing of the manuscript; Obtaining, analyzing and interpreting the data; Effective participation in research orientation; Critical review of the literature; Critical review of the manuscript

Javier Fernández $\quad$ (iD) ORCID 0000-0002-7110-3600

Approval of the final version of the manuscript; Elaboration and writing of the manuscript; Obtaining, analyzing and interpreting the data; Effective participation in research orientation; Intellectual participation in propaedeutic and/or therapeutic conduct of the cases studied; Critical review of the literature; Critical review of the manuscript

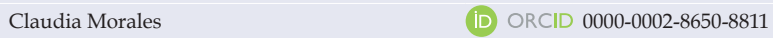

Obtaining, analyzing and interpreting the data; Intellectual participation in propaedeutic and/or therapeutic conduct of the cases studied
}

How to cite this article: Vargas P, Giacaman P, Fernández J, Morales C. Bullous pemphigoid associated with psoriasis: a good response to methotrexate. An Bras Dermatol. 2019;94(2):224-6. 